\begin{tabular}{|l|l|}
\hline VB-2 & 525 \\
\hline
\end{tabular}

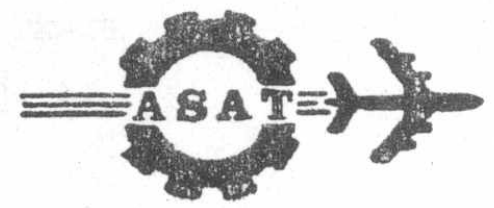

MILITARY TECHNICAL COLLEGE

CAIRO - EGYPT

\title{
A MODAL ANALYSIS OF A VIBRATING \\ SANDWICH CANTILEVER BEAM
}

\author{
S.H. Farghaly*
}

\begin{abstract}
An identification of the modal deflections, bending moments and strains for a three layer sandwich cantilever beam is presented in this paper. The analysis covers the first three vibrational modes for elastic cores with relatively thin face layers considering different shear and geometrical parameters. Both antinodal transverse and longitudinal strains and their locations are fully investigated. Nodal and peak points are specified for the three vibrational modes. These information provide proper design selection of different dimensions and operational dynamic properties.
\end{abstract}

* Assistant Professor, Mechanical Design Department, Faculty of Engineering and Technology, MATARIA, Helwan University, Cairo, Egypt. 


\section{INTRODUCTION}

For vibration control in machines and structures the resonance has to be avoided if not the amplitude of vibration at resonance has to be minimized. In many modern machinery and industrial applications, vibration excitation is encountered over a wide range of frequencies. Damped sandwich beams have recently acquired considerable importance for vibration response reduction in aircraft, spacecraft structures and related aerospace technology. When the structural masses are important, the undamped sandwich beam is frequently used. For example, sandwich structures are found in aeroplane wings and in the parts of structures which protect the casing of the equipment. In addition, for acoustical environment in aerospace structures, the honeycombed cores are considered the most light to damp the acoustical pressures.

The sandwich beam consists of two elastic external faces which are usually made of alluminium, stainaless steel, separated by a homogeneous or honeycombed core made of alluminium or fiberglass. In this configuration the normal forces due to bending are absorbed by the external layers and the shear load by the core. The three layers of the beam are bonded together by an adhesive material such as araldite. The probelem of fundamental mode analysis for bending vibrations of undamped sandwich cantilever beam is presented by Mead[1].

In this paper, the modal analysis of flexurally vibrating sandwich cantilever beam is presented. The first three modes of vibrations for, deflections, bending and longitudinal displacements are investigated. In addition, nodal and antinodal points, and the strains due to bending and the associated longitudinal deformations are studied.

\section{METHOD OF SOLUTION}

Important assumptions are usually considered by many investigators to simplify the theoritical analysis for the multilayered beam[2-3]. These include, the strain energy due to bending and longitudinal deformation of the elastic faces and that due to shear deformation of the core.

The equation of motion of the sandwich beam element shown in Fig. I with undamped core material is reported by [2-5] and may be written as

$$
\frac{\partial^{6} v}{\partial x^{6}}-X(1+Y) \frac{\partial^{4} v}{\partial x^{4}}+\frac{m}{B}\left(\frac{\partial^{4} v}{\partial^{2} x \partial^{2} t}-X \frac{\partial^{2} v}{\partial t^{2}}\right)=0
$$

where

$$
\begin{aligned}
v(\bar{x}, t) & =\quad V(x) \text { exp. } j \Omega t \text { for harmonic vibrations } \\
X & =\text { Shear parameter }=G_{2} L^{2} \bar{K} / 2 H_{2} K_{1} \\
Y & =\text { Geometrical parameter }=C^{2} K_{1} / B \bar{K} \\
m & =\text { Total mass of beam per unit length } \\
B & =\text { Bending stiffness }=E_{1} I_{1}+E_{3} I_{3}
\end{aligned}
$$


Introducing the dimension-less modal frequency parameter $a_{n s}$ we get

$$
a_{n s}=\omega_{n} \sqrt{m L^{4} / B}
$$

and substituting eq. (2) and eq. (3) into eq. (1) yields

$$
V^{V I}-X(1+Y) V^{I V}-a^{2} n s\left(V^{I I}-X V\right)=0
$$

The solution of the form

$$
V(x)=\sum_{i=1}^{6} A_{i} \exp \cdot j r_{i} x
$$

can be assumed. Substitution of eq. (5) into eq. (4) yield the following six order characteristic equation

$$
Z_{n}^{3}-X(1+Y) Z_{n}^{2}-a_{n s}\left(X+Z_{n}\right)=0
$$

where $z_{n}=r^{2}$

For a specific design problem the boundary conditions are known and eq. (5) can be written in matrix form as

$$
\left[a_{i j}\right]\left\lceil A_{j}\right]=[0]
$$

where $a_{i j}$ are the elements of the matrix which depend on the specific boundary conditions [1]. For a nontrivial solution, the determinant of the square matrix in eq. (7) must vanish. A root search routine is employed to find the desired values of $a_{\text {ns }}$ to satisfy the above equations. The corresponding mode shape is found by setting the coefficient $A_{1}=1$, and then computing the coefficient $\mathrm{A}_{2}$ to $\mathrm{A}_{6}$ by using the Gausian elimination technique[6]. Finally, the use of the values of $A_{i}$ gives the mode shapes and the subsequent significant analyses. The modal bending and longitudinal displacement can be written as follows:

$$
\begin{aligned}
& M=B V^{I I} \\
& U=\frac{B}{X^{2} C K_{1} L}\left[V^{V}-X Y V^{I I I}-\left(a_{n s}^{2}+X^{2} Y\right) V^{I}\right]
\end{aligned}
$$
Both the modal strain due to bending and the longitudinal displacement can
be expressed as:

$$
b=\frac{M C}{B}, \quad \epsilon_{1}=\frac{d u}{d x}
$$

\section{COMPUTATIONAL RESUI.TS AND DISCUSSION}

The computational results of the positive roots of the chracteristics equation are reported in Fig. 2. At small and large values of $\mathrm{X}$, the positive roots are approaching the first eigenvalue of the homogeneous cantilever beam (1.875)[1].

For the second and third mode (M2 and M3), some features of the positive roots are observed. These roots are approaching the second and third eigenvalues 
of the homogeneous cantilever beam $(4.694,7.85)$ respectively, for smaller and larger values of $\mathrm{X}$ as it is clearly shown. With reference to Figs. 3 modal frequency parameters are plotted for th me previous range of the geometrical parameters $X$. The modal frequency parameters are approaching the exact value of the homogeneous cantilever beam at smaller values of $\mathrm{X}$. For larger values, these parameters increase as $\mathrm{Y}$ iancreases for the three vibrational modes. The first three modal deflections, bendings, displacements and the anti-nodal points in which the computed parameter has its maximum value along the sandwich beam span, are plotted as a function of both the shear parameter $\mathrm{X}$ and the geometrical parameter $\mathrm{Y}$. The anti-nodal and peak points for both the modal deflection and bending are reported in Fig. 4 and 5 for specific value of $\mathrm{X}$ and $\mathrm{Y}$. The anti-nodal modal bending plots shown in Figs. 6 tend to increase with an increase in the geometrical parameter $Y$, as well as the $\mathrm{X}$ parameter. The variations of the anti-nodal deflection as a function of both $\mathrm{X}$ and $\mathrm{Y}$ are shown in Figs. 7. These figures may be used as a design chart for selecting minimal modal deflections point for different $X$ and $Y$ parameters. Anti-nodal modal displacements illustrated in Figs. 8 show increasing values with a high rate with the shear parameter specially for higher modes.

\section{CONCLUSION}

A complete theoretical analysis has been presented for a sandwich cantilever beam. This includes, the positive roots of characteristic equation, modal frequency parameters, modal deflections, bending, displacements and their antinodal points for a wide range of the geometrical and shear parameters. The analysis covers three vibrational modes with soft and stiff beam core (for 0.01 $<\mathrm{X}<200)$. The comprehensive plots which are deduced from the present work can be used as design charts for such a vital widely used structural system.

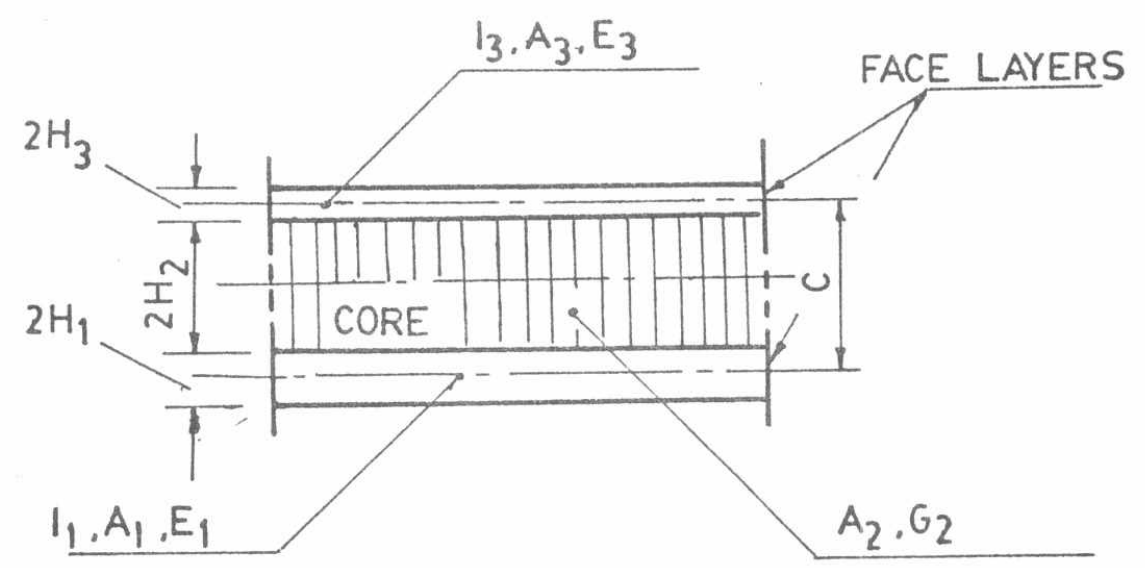

Fig. 1 Sandwich beam element 

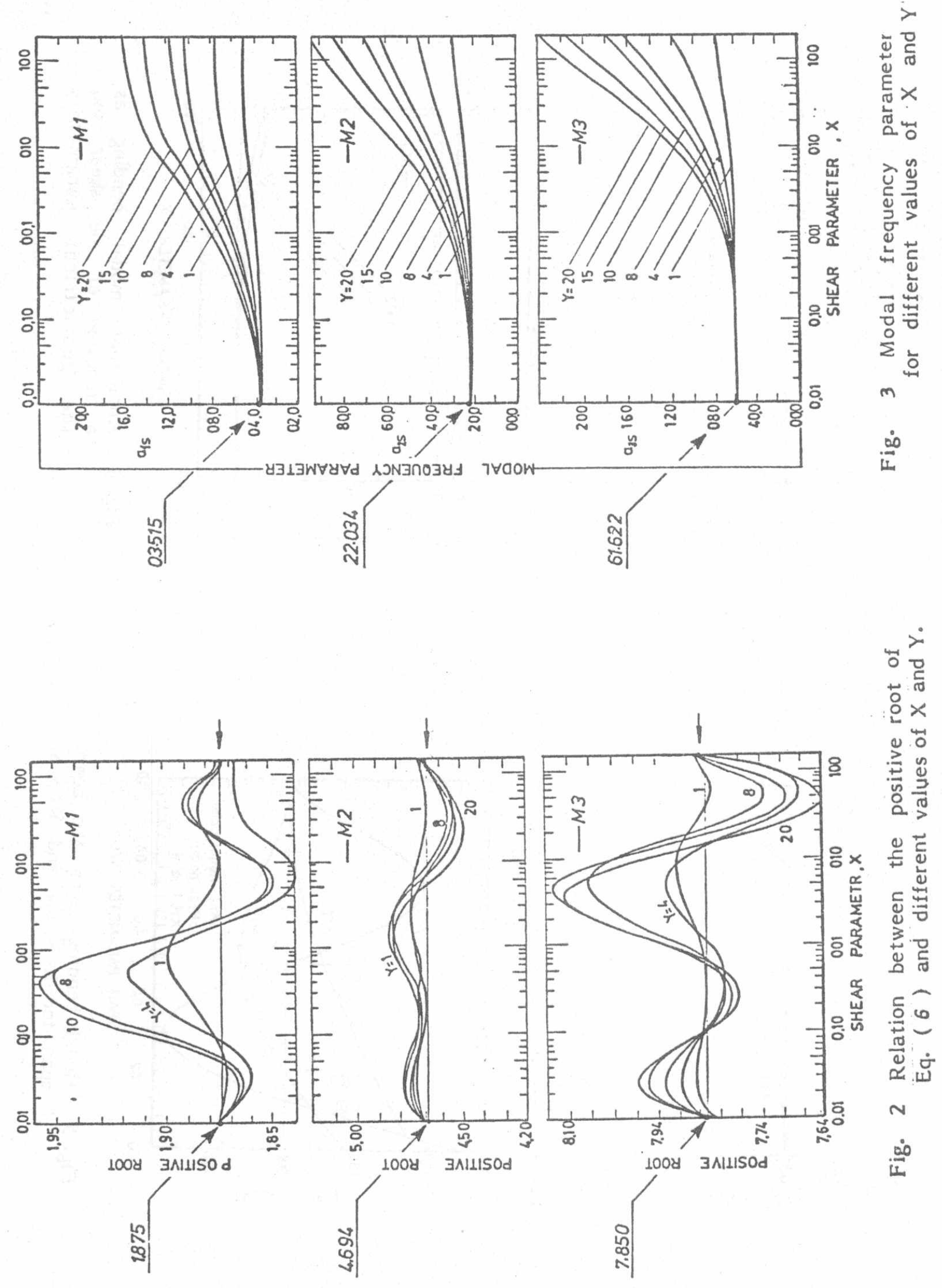

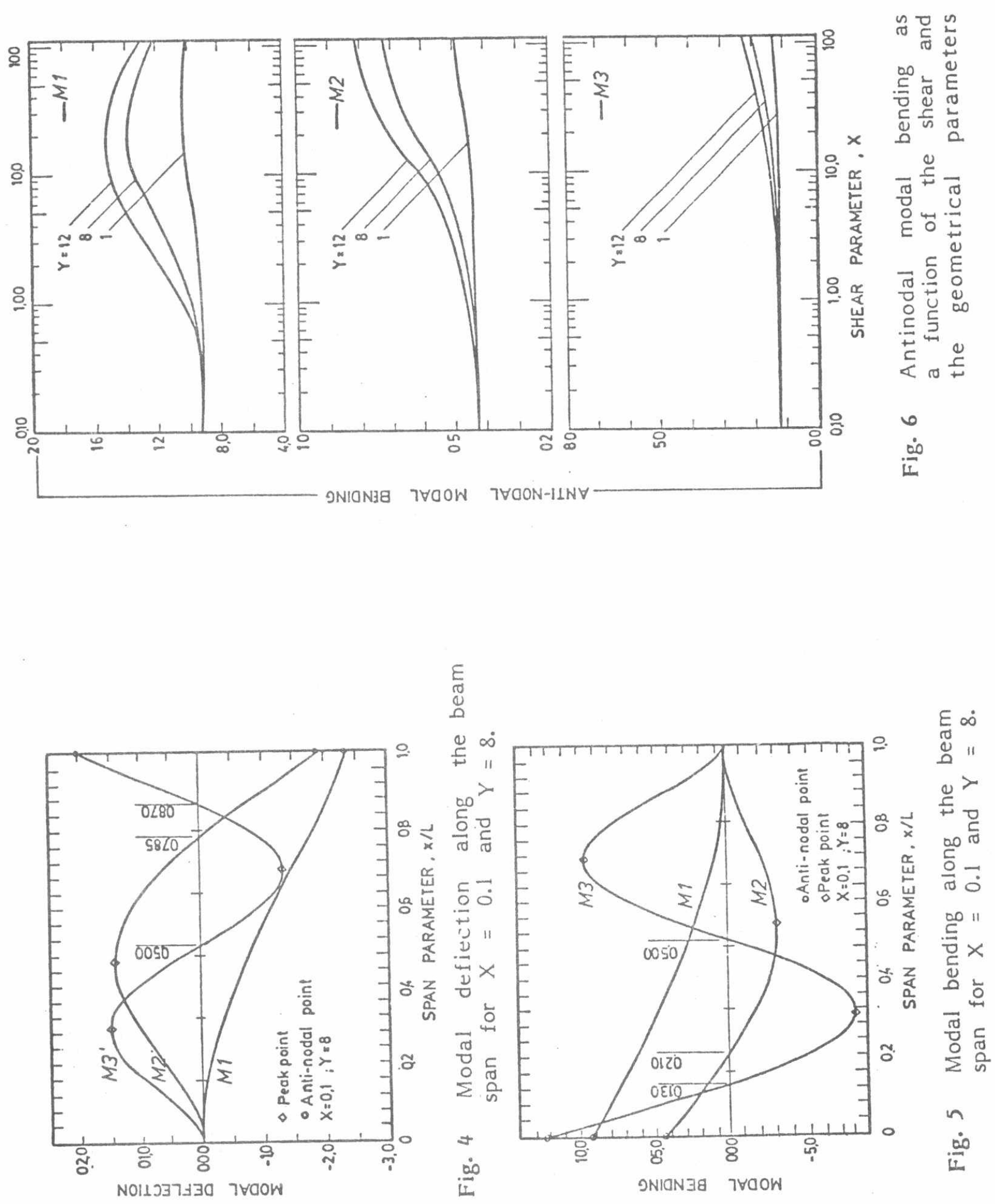

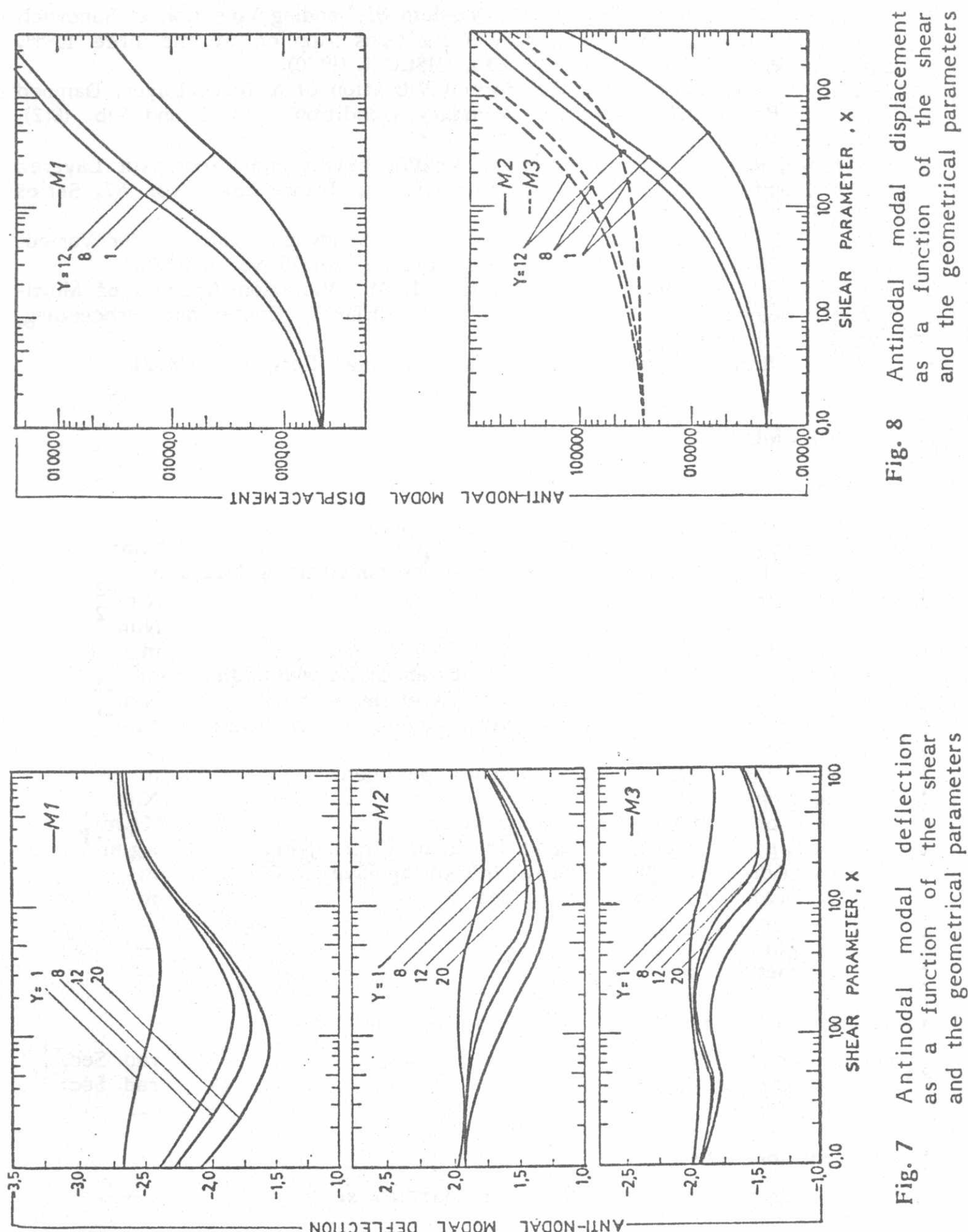

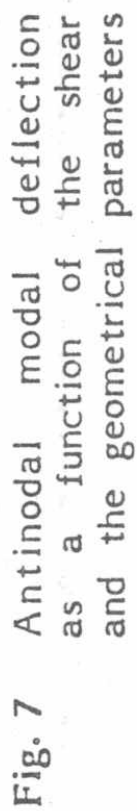

N011037 330 7V0OW TVOON-IINY 


\section{REFERENCES}

1. Mead, D.J. and Markus, S. "On the Problem of Bending Vibration of Sandwich Cantilevers with Various Boundary Conditions Applied at the Free End", STROJNICKY CASOPIS, ROCNIN XXI, CISLO 1, (1970).

2. Mead, D.J. and Markus, S. "The Forced Vibration of a Three Layer, Damped Sandwich Beam with Arbitrary Boundary Conditions. J. Sound Vib. 10(2), Sept. (1969).

3. DiTaranto, R.A. "Theory of Vibratory Bending Elastic and Viscoelastic Layered Finite Length Beams. J. Appl. Mechanics, 32, Trans. ASME vol. 87, Series E, (1965).

4. Rao, D.K., "Frequency and Loss Factors of Sandwich Beams Under Various Boundary Conditions", J. of Mech. Eng. Science, Vol 20 No. 5, (1978).

5. Farghaly, S.H., El-Sherif, F.S. and Ismail, M. "Vibration Analysis of Multilayered Beams with Viscoelastic Core", PEDAC, Conference Proceeding, Vol. II, pp. 933-942, (1983).

6. Rao, S.S., "Finite Element Method in Engineering", Pergamon, (1982).

\section{NOMENCLATURE}

\section{Latin Letters}

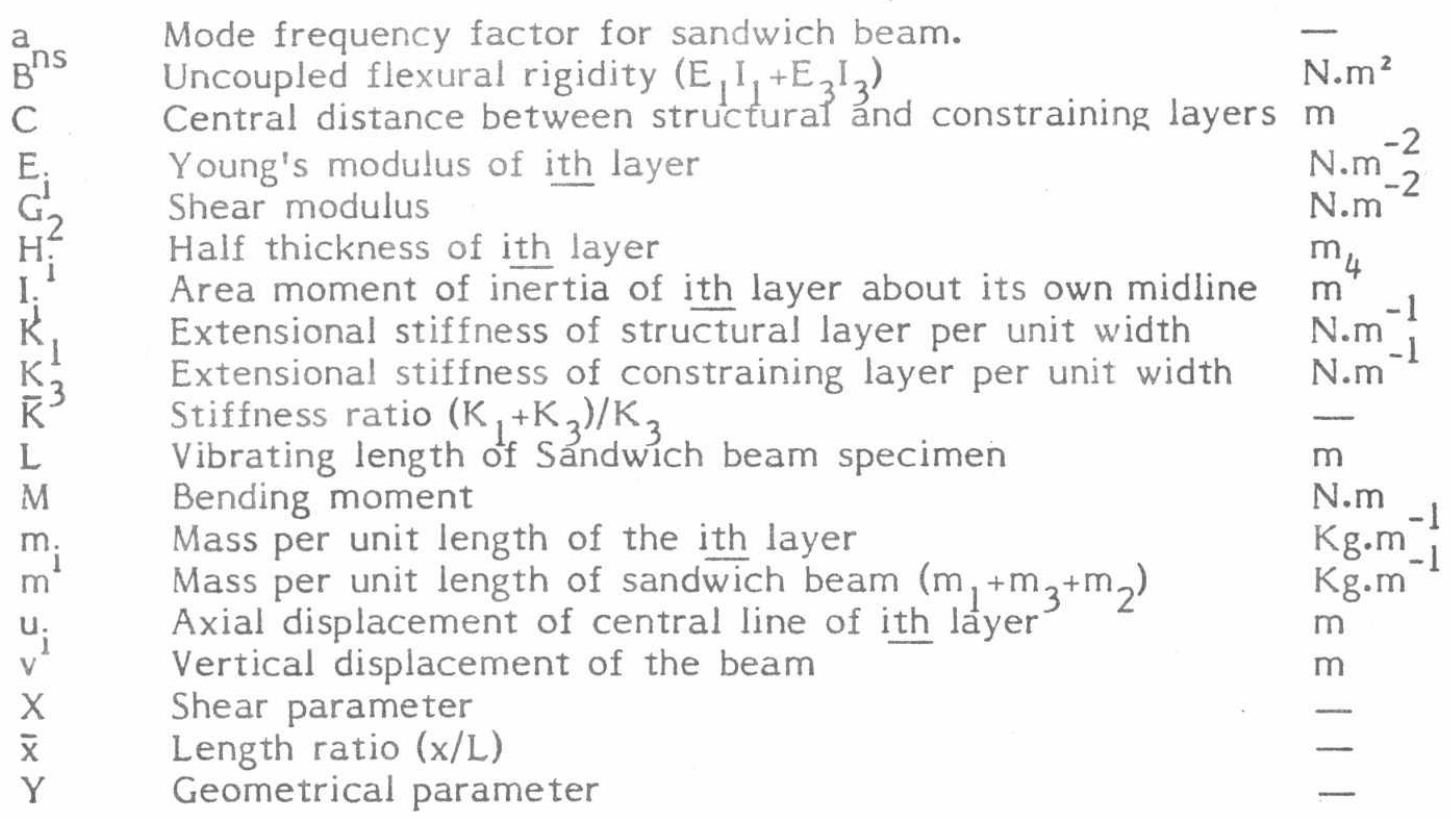

\section{Greek Letters}

$\begin{array}{ll}\omega & \text { Resonant frequency at mode } n \\ \Omega^{n} & \text { Excitation frequency } \\ \epsilon & \text { Strain }\end{array}$

\section{Superscripts for letters}

I Designates the derivation with respect to, $x$. 